\title{
The Interaction of a New Motor Skill and an Old One: H-Reflex Conditioning and Locomotion in Rats
}

\author{
Yi Chen, ${ }^{1,2}$ Xiang Yang Chen, ${ }^{1}$ Lyn B. Jakeman, ${ }^{2}$ Gerwin Schalk, ${ }^{1}$ Bradford T. Stokes, ${ }^{2}$ and Jonathan R. Wolpaw ${ }^{1}$ \\ ${ }^{1}$ Laboratory of Nervous System Disorders, Wadsworth Center, New York State Department of Health and State University of New York, Albany, New York \\ 12201, and ${ }^{2}$ Department of Physiology and Cell Biology, The Ohio State University, Columbus, Ohio 43210
}

New and old motor skills can interfere with each other or interact in other ways. Because each skill entails a distributed pattern of activity-dependent plasticity, investigation of their interactions is facilitated by simple models. In a well characterized model of simple learning, rats and monkeys gradually change the size of the H-reflex, the electrical analog of the spinal stretch reflex. This study evaluates in normal rats the interactions of this new skill of H-reflex conditioning with the old well established skill of overground locomotion.

In rats in which the soleus H-reflex elicited in the conditioning protocol (i.e., the conditioning H-reflex) had been decreased by down-conditioning, the H-reflexes elicited during the stance and swing phases of locomotion (i.e., the locomotor H-reflexes) were also smaller. Similarly, in rats in which the conditioning H-reflex had been increased by up-conditioning, the locomotor H-reflexes were also larger.

Soleus H-reflex conditioning did not affect the duration, length, or right/left symmetry of the step cycle. However, the conditioned change in the stance H-reflex was positively correlated with change in the amplitude of the soleus locomotor burst, and the correlation was consistent with current estimates of the contribution of primary afferent input to the burst.

Although H-reflex conditioning and locomotion did not interfere with each other, H-reflex conditioning did affect how locomotion was produced: it changed soleus burst amplitude and may have induced compensatory changes in the activity of other muscles. These results illustrate and clarify the subtlety and complexity of skill interactions. They also suggest that H-reflex conditioning might be used to improve the abnormal locomotion produced by spinal cord injury or other disorders of supraspinal control.

Key words: H-reflex conditioning; spinal cord plasticity; motor control; learning; memory consolidation; locomotion; rehabilitation

\section{Introduction}

The nervous system maintains a broad repertoire of adaptive behaviors acquired through practice, commonly referred to as skills (Compact Oxford English Dictionary, 1993). New skills may interfere, or interact in other ways, with old ones. These interactions are often addressed in terms of unitary concepts of memory consolidation, reactivation, and interference (Shadmehr and Holcomb, 1997; Krakauer et al., 1999; Goedert and Willingham, 2002; Wigmore et al., 2002; Walker et al., 2003; Caithness et al., 2004). However, it is now clear that even the simplest skills involve complex distributed patterns of activitydependent plasticity (Wolpaw and Lee, 1989; Carrier et al., 1997; Cohen et al., 1997; Lieb and Frost, 1997; Thompson et al., 1997; Whelan and Pearson, 1997; Lisberger, 1998; Garcia et al., 1999; Medina et al., 2000, 2002; Hansel et al., 2001; King et al., 2001;

Received Feb. 17, 2005; revised June 9, 2005; accepted June 10, 2005

This work was supported by National Institutes of Health Grants HD36020 (X.Y.C.), NS22189 (J.R.W.), and NS37321 (B.T.S.), The Christopher Reeve Paralysis Foundation (X.Y.C.), and The International Spinal Research Trust (J.R.W.). It constitutes a part of Y.C.'s doctoral thesis to be presented at The Ohio State University. We thank Lu Chen and Rongliang Liu for excellent technical assistance and Drs. John A. Buford, Jonathan S. Carp, and Elizabeth Winter Wolpaw for valuable advice and comments on this manuscript.

Correspondence should be addressed to Xiang Yang Chen, Laboratory of Nervous System Disorders, Wadsworth Center, New York State Department of Health, P.0. Box 509, Albany, NY 12201-0509. E-mail: chenx@wadsworth.org.

DOI:10.1523/JNEUROSCI.1684-05.2005

Copyright $\odot 2005$ Society for Neuroscience $\quad$ 0270-6474/05/256898-09\$15.00/0
Wolpaw and Tennissen, 2001; Carey and Lisberger, 2002; van Alphen and De Zeeuw, 2002; Wolpaw, 2002; Blazquez et al., 2003). A new skill may involve different kinds of plasticity that occur at different sites at different rates and that differently affect (and are differently affected by) the similarly complex plasticity associated with an old skill. Thus, the study of interactions between skills can benefit from simple learning models that allow these interactions to be explored on the level of their neuronal and synaptic mechanisms.

The spinal cord provides a unique opportunity for such explorations. As the final common pathway for neuromuscular behaviors, its motoneurons, interneurons, and their connections serve the entire behavioral repertoire and, like the rest of the CNS, undergo activity-dependent plasticity throughout life (for review, see Wolpaw and Tennissen, 2001). During early development and during skill acquisition later on, descending input from the brain combines with peripheral input to change the spinal cord so as to ensure satisfactory performance of all the many behaviors that issue from it. At the same time, the relative simplicity and experimental accessibility of the spinal cord and the tracts that connect it to the brain facilitate localization and exploration of the spinal and supraspinal plasticity associated with the acquisition of a new skill.

These advantages are best exemplified by the spinal stretch reflex (SSR) (i.e., the tendon jerk) and its electrical analog, the 
H-reflex, which are the simplest motor behaviors. They are mediated primarily by a two-neuron, monosynaptic pathway consisting of the primary afferent (i.e., group Ia or large group II) fiber, its synapse on the motoneuron, and the motoneuron itself (Magladery et al., 1951; Matthews, 1972; Baldissera et al., 1981; Henneman and Mendell, 1981; Brown, 1984). Because it is influenced by descending activity from the brain, this pathway can be operantly conditioned. In response to an operant conditioning protocol, monkeys (Wolpaw et al., 1983a; Wolpaw, 1987), humans (Evatt et al., 1989), and rats (Chen and Wolpaw, 1995) can gradually decrease [i.e., down-conditioning mode (HRdown)] or increase [i.e., up-conditioning mode (HRup)] the SSR or the H-reflex. Acquisition of these simple motor skills (i.e., a smaller or larger H-reflex), which occurs over days and weeks of practice (i.e., of exposure to the conditioning protocol), is associated with complex patterns of activity-dependent spinal and supraspinal plasticity that include changes in motoneuron firing threshold and conduction velocity in several different synaptic terminal populations on the motoneuron and probably in spinal interneurons and supraspinal regions as well (Wolpaw, 2001; Wolpaw and Tennissen, 2001).

Because spinal motoneurons and interneurons mediate all motor behaviors, the plasticity directly responsible for $\mathrm{H}$-reflex change is likely to affect behaviors other than the H-reflex. For example, the change in motoneuron response to primary afferent input caused by H-reflex conditioning could affect locomotion, and the change in motoneuron firing threshold could affect essentially every behavior.

This study explores in normal rats the interactions of $\mathrm{H}$-reflex conditioning with locomotion, an important, well characterized, and quantifiable skill in which the primary afferent excitation responsible for the H-reflex plays a major role (Grillner, 1981; Yang et al., 1991; Pearson, 1993; Bennett et al., 1996; Stein et al., 2000). It asks: (1) whether the H-reflex change created by conditioning is affected by locomotion; (2) whether H-reflex conditioning affects locomotion, as assessed by the duration and symmetry of the step cycle; and (3) whether H-reflex conditioning affects the CNS activity responsible for locomotion, as assessed by the EMG burst during the stance phase. The results illustrate and clarify the interactions of new and old motor skills.

\section{Materials and Methods}

Subjects were 18 Sprague Dawley rats (14 males, 4 females; $259-403$ g at the beginning of the study). Evaluation of the data from previous studies of 135 normal rats (82 males and 53 females) (Chen and Wolpaw, 1995, 1996, 1997, 2002; Chen et al., 1996, 1999, 2001a,b, 2002a,b, 2003; our unpublished data) indicated that none of the physiological variables involved in this study (e.g., background EMG level, H-reflex size, M-response size) differs significantly between males and females. All procedures satisfied the Guide for the Care and Use of Laboratory Animals of the Institute of Laboratory Animal Resources, Commission on Life Sciences, National Research Council [National Academy Press, Washington, DC (1996)] and had been reviewed and approved by the Institutional Animal Care and Use Committee of the Wadsworth Center. The procedures for animal preparation, H-reflex conditioning, and data collection in freely moving rats have been described in detail previously (Chen and Wolpaw, 1995, 1996, 1997, 2002; Chen et al., 1996, 1999, 2001a) and are summarized here. The procedure for data collection during treadmill locomotion is described fully.

Animal preparation and environment. Under general anesthesia (ketamine $\mathrm{HCl}, 80 \mathrm{mg} / \mathrm{kg}$, i.p.; xylazine, $10 \mathrm{mg} / \mathrm{kg}$, i.p.), each rat was implanted with chronic stimulating and recording electrodes. To elicit the H-reflex, a silicone rubber nerve cuff containing a pair of stainless-steel multistranded fine-wire electrodes was placed on the right posterior tibial nerve just above the triceps surae branches. To record soleus EMG activity, pairs of fine-wire electrodes with the final $0.5 \mathrm{~cm}$ stripped were placed in the right and left soleus muscles (only right soleus in four rats). The Teflon-coated wires from the nerve cuff and the muscles passed subcutaneously to a connector plug mounted on the skull with stainlesssteel screws and dental cement. Immediately after surgery, the rat was placed under a heating lamp and given an analgesic (Demerol, $0.2 \mathrm{mg}$, i.m.). Once awake, it received a second dose of analgesic and was returned to its cage and allowed to eat and drink freely.

Throughout the study, the rat lived in a standard rat cage with a $40 \mathrm{~cm}$ flexible cable attached to the skull plug. The cable, which allowed the animal to move freely in the cage, carried the wires from the electrodes to a commutator above the cage that connected to EMG amplifiers and a nerve-cuff stimulation unit. The rat had ad libitum access to water throughout. During H-reflex conditioning, it received most of its food by performing the task described below. Animal well being was carefully checked several times each day, and body weight was measured weekly. Laboratory lights were dimmed from 9:00 P.M. to 6:00 A.M. daily.

The H-reflex conditioning protocol. Data collection began at least $20 \mathrm{~d}$ after the implantation surgery. A computer system continuously monitored $(24 \mathrm{~h} / \mathrm{d})$ the right soleus EMG and controlled two outputs: the nerve-cuff stimulus and the reward (i.e., a $20 \mathrm{mg}$ food pellet). If the absolute value of background (i.e., ongoing) EMG (i.e., equivalent to the full-wave rectified value) remained within a specified range for a randomly varying $2.3-2.7 \mathrm{~s}$ period, a stimulus pulse (typically $0.5 \mathrm{~ms}$ in duration) was delivered by the nerve cuff. Pulse amplitude was initially set just above M-response (i.e., the direct muscle response to nerve stimulation) threshold and then continuously and automatically adjusted by the computer to maintain M-response size unchanged throughout the whole period of data collection (which ensured that the effective strength of the nerve-cuff stimulus did not change). Under the control mode, the computer simply measured the absolute value of soleus EMG for $50 \mathrm{~ms}$ after the stimulus. Under the HRdown or HRup conditioning mode, a food reward was dispensed $200 \mathrm{~ms}$ after nerve stimulation if EMG amplitude in the H-reflex interval (e.g., 5.5-9.5 ms after stimulation) was below (HRdown mode) or above (HRup mode) a criterion value. In the course of its normal activity, the animal usually satisfied the background EMG requirement, and thus received nerve-cuff stimulation, 2500-8000 times per day. H-reflex size was calculated as average EMG amplitude in the H-reflex interval minus average background EMG amplitude at the time of stimulation and was expressed in units of average background EMG amplitude. This H-reflex elicited in the conditioning protocol is designated the conditioning $\mathrm{H}$-reflex to distinguish it from the H-reflexes elicited during the stance and swing phases of locomotion, which are designated the locomotor H-reflexes.

Figure $1 A$ summarizes the study design. Data were collected under the control mode for $20 \mathrm{~d}$ to determine the initial size of the animal's conditioning H-reflex. It was then exposed to the HRdown or HRup mode for $50 \mathrm{~d}$. To determine the final effect on conditioning H-reflex size of HRdown or HRup mode exposure, average $\mathrm{H}$-reflex size for the final $10 \mathrm{~d}$ of the exposure was calculated as percentage of initial (i.e., average of final 10 control-mode days) H-reflex size. As in the past, successful conditioning was defined as a change of $\geq 20 \%$ in the correct direction (Wolpaw et al., 1993; Chen and Wolpaw, 1995).

Treadmill locomotion and the locomotor $H$-reflexes. As indicated in Figure $1 \mathrm{~A}$, before the implantation surgery, each rat learned in one to two sessions (one per day; 10-20 min walking per session) to walk quadrupedally on a motor-driven treadmill (Eco 3/6 treadmill; Columbus Instruments, Columbus, $\mathrm{OH}$ ) at a speed of $9-16 \mathrm{~m} / \mathrm{min}$ (Burghardt et al., 2004). During this training, the rat was motivated primarily by food reward (bread or cereal). In a few rats, this was supplemented early in training by a weak $(0.69 \mathrm{~mA}, 0.2 \mathrm{~s})$ electric stimulus from a metal grid just behind the posterior end of the treadmill. This minimal aversive stimulus caused no vocalization or other evidence of significant distress and was administered only once or twice per rat. The preimplantation training was effective: when they were placed on the treadmill for actual locomotor data collection later on, rats typically walked immediately. Because rat $\mathrm{H}$-reflex size displays a diurnal variation (opposite in phase to that found in monkeys) (Chen et al., 2002b), locomotor data from each rat were always collected at the same time of day. 
Locomotor data were collected in one treadmill session during the 20 control-mode days and in another treadmill session during the final $10 \mathrm{~d}$ of exposure to the HRdown or HRup mode (Fig. $1 A$ ). For these sessions, the rat was transferred from its cage to the treadmill, and the head-mounted cable was connected to a commutator above the treadmill. For each rat, treadmill speed was the same before and after conditioning. During locomotion, EMG was recorded continuously $(0.1-1.0 \mathrm{kHz}$ bandpass) from right and left (14 rats) [or only right (four rats)] soleus muscles and digitized $(4.0 \mathrm{kHz})$ and stored by computer. Figure $1 B$ shows EMG recorded from right and left soleus muscles during locomotion.

In each treadmill session for each rat, data were collected under three different conditions. In conditions $\mathrm{A}$ and $\mathrm{B}$, whenever right soleus EMG satisfied defined criteria (see below), a stimulus pulse (typically $0.5 \mathrm{~ms}$ in duration and kept just above M-response threshold as described above) was delivered by the nerve cuff to elicit the right soleus $\mathrm{H}$-reflex. In condition $\mathrm{A}$ (Fig. 2A), the stimulus was delivered when the right soleus EMG remained in a specified high range (and the left soleus EMG remained in a specified low range) for $200 \mathrm{~ms}$. These criteria placed the stimulus past the middle of the right soleus locomotor burst and thus in the later part of the right stance phase of the step cycle. In condition B (Fig. 2 B), the stimulus was delivered when the right soleus EMG remained in a specified low range (and the left soleus EMG remained in a specified high range) for $200 \mathrm{~ms}$. These criteria placed the stimulus late in the right swing phase of the step cycle. Condition $\mathrm{C}$ had the same EMG criteria as condition A. The difference was that no stimulus was actually delivered to the nerve cuff, although the computer continued to generate and record the stimulus trigger. Conditions A and B were used to study the locomotor $\mathrm{H}$-reflexes, that is, the $\mathrm{H}$-reflexes during the stance and swing phases of locomotion, respectively. Condition $\mathrm{C}$ examined right and left soleus EMG during undisturbed locomotion. Approximately five minutes of data were collected under each condition (beginning with condition C) from each rat in each session and were stored for later analysis.

To establish the relationship between the soleus locomotor burst and the stance phase of locomotion, a video camera recorded (at 60 frames per second) treadmill locomotion from the right side while EMG data were collected under condition C (i.e., no nerve-cuff stimulation) in four rats. A custom off-line analysis program used the recorded video to determine for each step cycle the time during which part or all of the right hindfoot was within $1 \mathrm{~mm}$ of the treadmill surface. This time was defined as the right stance period, and its timing was compared with that of the EMG burst from the right soleus. Figure $1 C$ illustrates the typically close relationship between the onsets of the soleus EMG burst and the stance phase of locomotion.

Perfusion and postmortem examination. After data collection was completed for this study, each rat was used in one of several other studies of the long-term effects of H-reflex conditioning, after which it received an overdose of sodium pentobarbital (intraperitoneal) and was perfused through the heart with saline followed by $4 \%$ paraformaldehyde solution. The nerve cuff, the EMG electrodes, and the tibial nerve were examined, and the soleus muscles of both sides were removed and weighed.

Analysis of $\mathrm{H}$-reflexes during locomotion. The EMG recorded under conditions $\mathrm{A}$ and $\mathrm{B}$ was rectified and used to measure $\mathrm{H}$-reflexes during the stance and swing phases of locomotion, respectively, as illustrated in Figure 2. In these off-line analyses, the computer triggered on the stim-
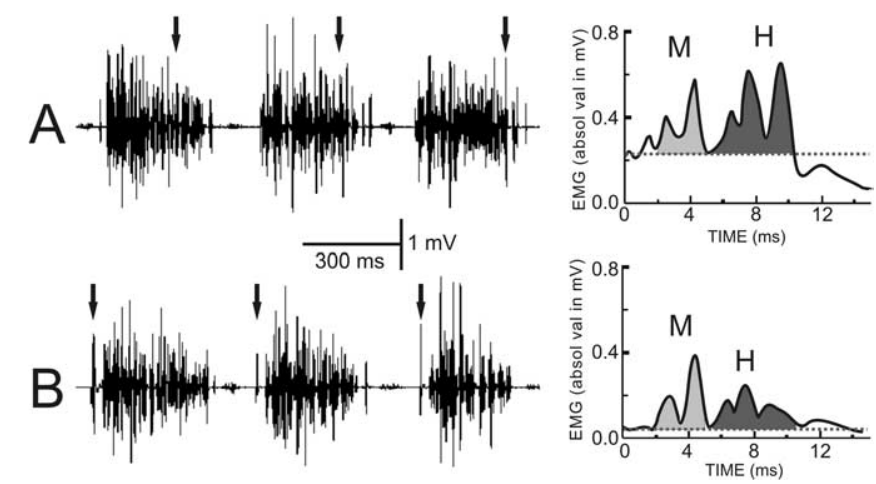

Figure 2. Locomotor H-reflexes: elicitation of the right soleus $\mathrm{H}$-reflex during the stance and swing phases of the step cycle. $\boldsymbol{A}$, Left, Stimulus (arrow) during the right soleus burst elicits the stance H-reflex. Right, Average absolute value of right soleus EMG after stimulation (at $0 \mathrm{~ms}$ ) during stance. The dotted line indicates the background EMG level at the time of stimulation, and the $M$ response and $H$-reflex are shaded. $\boldsymbol{B}$, Left, Stimulus (arrow) when right soleus EMG is low elicits the swing $\mathrm{H}$-reflex. Right, Average absolute value of right soleus EMG after stimulation during swing. Note that M-response size (and thus effective stimulus strength) is similar in the stance and swing phases, whereas the H-reflex is much larger during the stance phase when background EMG is much higher. absol val, Absolute value.

ulus and digitized the EMG for up to $500 \mathrm{~ms}$ before and after stimulation. It then averaged those trials for which background EMG amplitude (i.e., EMG amplitude for the $20 \mathrm{~ms}$ immediately before the stimulus) and M-response size satisfied specified criteria. These criteria ensured that the H-reflexes before and after conditioning were measured at the same 
background EMG amplitude and M-response size and thereby allowed us to assess the effect of conditioning on the H-reflexes. That is, the criteria applied to the stance H-reflex data ensured that the background EMG amplitude and M-response size for the stance H-reflex measured before conditioning were the same as for the stance H-reflex measured after conditioning, and the criteria applied to the swing H-reflex data ensured that the background EMG amplitude and M-response size for the swing $\mathrm{H}$-reflex measured before conditioning were the same as for the swing H-reflex measured after conditioning. (Soleus burst amplitudes typically varied considerably across step cycles. Thus, even when $\mathrm{H}$-reflex conditioning changed the average burst amplitude, there was enough overlap between burst amplitudes before and after conditioning so that these criteria were able to ensure that locomotor H-reflex measurements before and after conditioning were derived only from step cycles for which soleus burst amplitudes were comparable.)

Just as for the conditioning H-reflexes, locomotor $\mathrm{H}$-reflex sizes were calculated as average EMG amplitude in the H-reflex interval minus average background EMG amplitude at the time of stimulation and were expressed in units of average background EMG amplitude. The impacts of down-conditioning and up-conditioning on these reflexes were assessed by comparing the reflexes before and after conditioning by paired $t$ test.

Analysis of locomotion. To assess locomotion, the EMG recorded under condition C (i.e., no nerve-cuff stimulus) was rectified and low-pass filtered by a $50 \mathrm{~ms}$ running average. It was then used to assess the right and left soleus locomotor bursts, and these bursts were in turn used to assess step-cycle duration, length, and symmetry. Automated analysis identified the bursts by detecting the points at which soleus EMG reached (i.e., burst onset) and fell below (i.e., end of burst) $10 \%$ of its maximum value and then calculated for each session of each rat the average: stepcycle duration (time between right burst onsets in seconds), step-cycle length (treadmill speed in $\mathrm{cm} / \mathrm{s}$ times step-cycle duration in seconds), right and left burst durations (time from burst onset to offset), right and left burst amplitudes (total EMG area between burst onset and offset divided by burst duration), and step-cycle symmetry. Step-cycle symmetry was defined as the time from right burst onset to left burst onset divided by the time from right burst onset to the next right burst onset (i.e., the time of a full step cycle). Thus, a value of 0.5 indicates that the right/left timing of the step cycle, as assessed by the soleus bursts, was symmetrical. The impact of conditioning on these locomotor measures was assessed by the correlations (determined by linear regression) between changes in them and change in the stance H-reflex. We focused on the stance H-reflex because it directly reflects the impact of H-reflex conditioning on the soleus response to primary afferent input during the soleus contribution to locomotion (i.e., during the soleus burst).

\section{Results}

All rats remained healthy and active and continued to gain weight throughout the study. Body weight increased from 359 ( \pm 69 SD) $\mathrm{g}$ at the time of implantation surgery to $515( \pm 109) \mathrm{g}$ at the time of perfusion. At the end of study, soleus muscle weights (measured as percentage of body weight) were symmetrical and did not differ significantly between down-conditioned and upconditioned rats.

\section{Effects of conditioning on the conditioning H-reflex}

In magnitude of conditioning $\mathrm{H}$-reflex change and rate of success [i.e., H-reflex change $\geq 20 \%$ in the correct direction (Wolpaw et al., 1993; Chen and Wolpaw, 1995)], the results of the present study were similar to those of previous studies (Chen and Wolpaw, 1995, 1996; Chen et al., 1999, 2001b, 2002a; Carp et al., 2001). Conditioning was successful in 13 of the 18 rats [ 8 of 12 HRdown rats (five of the eight males and three of the four females) and in five of six HRup rats (all male)]. (As noted in Materials and Methods, males and females do not differ in any of the physiological variables assessed here.) In the successful HRdown rats, the conditioning H-reflex size fell to $50 \%$ ( $\pm 2 \% \mathrm{SE})$,

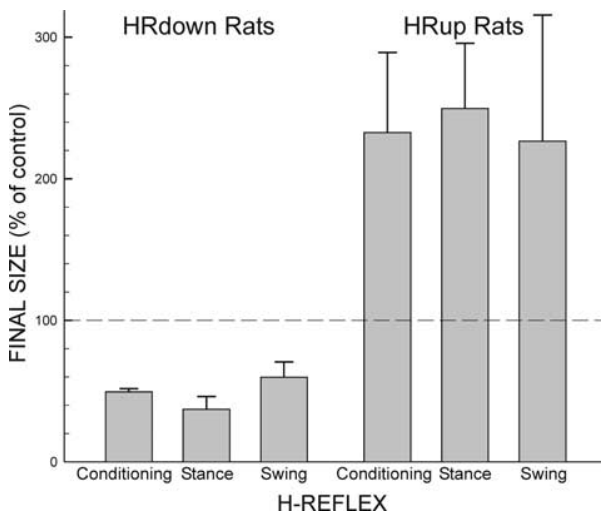

Figure 3. Effects of conditioning on the conditioning H-reflexes and the locomotor $\mathrm{H}$-reflexes. The average $\pm \mathrm{SE}$ final values of conditioning, stance, and swing $\mathrm{H}$-reflexes from successful HRdown and HRup rats are shown. The conditioning and locomotor H-reflexes are similarly decreased in the HRdown rats and similarly increased in the HRup rats.

and in the successful HRup rats, it rose to $232 \%$ ( $\pm 56 \% \mathrm{SE})$. In the remaining four HRdown rats and one HRup rat, the conditioning H-reflex remained within $20 \%$ of its control size. In all rats, background EMG and M-response size during measurement of the conditioning H-reflex remained stable throughout data collection.

\section{Effects of conditioning on the locomotor H-reflexes}

Successful HRdown or HRup conditioning produced comparable changes in the locomotor H-reflexes elicited during the stance and swing phases of locomotion. In the successful HRdown rats, $\mathrm{H}$-reflex size in the stance phase fell to $39 \%( \pm 8 \% \mathrm{SE})$ of its control value ( $p=0.0002$ by paired $t$ test), and H-reflex size in the swing phase fell to $59 \%$ ( $\pm 11 \% \mathrm{SE})(p=0.007)$. In the successful HRup rats, $\mathrm{H}$-reflex size in the stance phase rose to $252 \%$ ( $\pm 46 \%$ SE) $(p=0.03)$, and H-reflex size in the swing phase rose to $229 \%$ ( $\pm 87 \%$ SE) $(p=0.2)$. Figure 3 summarizes these results. Figure 4 shows protocol, stance-phase, and swingphase H-reflexes before and after conditioning from one HRdown rat and one HRup rat. After conditioning, the conditioning and locomotor H-reflexes are all smaller in the HRdown rat and larger in the HRup rat. Although M-response size differed across animals and across the conditioning, stance, and swing reflexes of individual animals (Fig. 4), these differences (which reflect interanimal differences in $\mathrm{M}$-response recruitment curves and other factors) did not alter the impact of H-reflex conditioning on H-reflex size.

In an ancillary effort to assess the likely impact of soleus $\mathrm{H}$-reflex conditioning on synergist muscles during locomotion, we evaluated unpublished gastrocnemius data gathered in previous studies of soleus H-reflex conditioning (Chen and Wolpaw, 1995, 1996, 1997, 2002; Chen et al., 1996, 1999, 2001a,b, 2002a,b, 2003; our unpublished data). In 17 rats in which the soleus $\mathrm{H}$-reflex was decreased (nine HRdown rats) or increased (eight HRup rats) by conditioning, the gastrocnemius H-reflex underwent a similar but lesser change. In the HRdown rats, it decreased $24 \%( \pm 17 \%$ SE) as much as the soleus H-reflex, and in the HRup rats, it increased $18 \%( \pm 8 \%)$ as much. These results are consistent with primate data on the muscular specificity of conditioning (Wolpaw et al., 1983b) and imply that the gastrocnemius locomotor H-reflexes were affected by conditioning in the same way as the soleus locomotor H-reflexes, but to a lesser degree.

The results from the unsuccessful HRdown rats were surprising. As noted above, in these four rats, the conditioning 

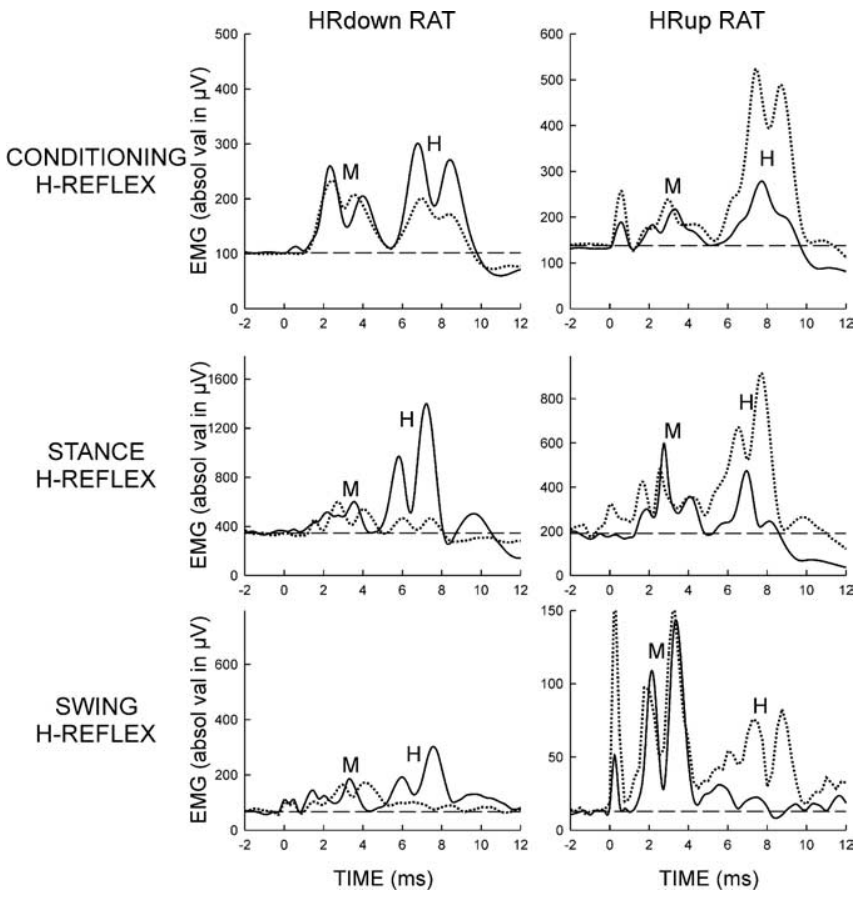

Figure 4. Conditioning and locomotor H-reflexes before (solid) and after (dotted) conditioning from an HRdown and an HRup rat. The conditioning $\mathrm{H}$-reflexes are each the average of a single day (at least 4000 trials), and the locomotor $\mathrm{H}$-reflexes are each the average of 315-531 trials obtained during the treadmill session. (In several traces, stimulus artifacts are present in the first millisecond after the stimulus.) After conditioning, the conditioning and locomotor $\mathrm{H}$-reflexes are smaller in the HRdown rat and larger in the HRup rat. absol val, Absolute value.

H-reflexes after conditioning were within $20 \%$ of their control values, and they averaged $99 \%$ ( $\pm 6 \% \mathrm{SE}$ ). In two of the four, the stance and swing $\mathrm{H}$-reflexes after conditioning were also within $20 \%$ of their control values. However, in the other two unsuccessful HRdown rats, the stance and swing H-reflexes were markedly larger after conditioning, rising to 358 and $126 \%$ in one rat and to 396 and $183 \%$ in the other. For one of these two rats, Figure 5 shows conditioning, stance, and swing $\mathrm{H}$-reflexes before and after conditioning. After attempted down-conditioning, the conditioning H-reflex is nearly the same as its control, whereas the locomotor H-reflexes are much larger than their controls.

\section{Effects of conditioning on locomotion}

Table 1 summarizes the impact of conditioning on locomotion. It shows, for successful HRdown and HRup rats, average \pm SE right and left soleus burst amplitudes and durations and average stepcycle duration and right/left symmetry after conditioning, expressed in terms of their control (i.e., preconditioning) values. It also shows the correlation of the change in each measure with the conditioned change in the stance H-reflex.

H-reflex conditioning affected the amplitude of the right soleus burst. This amplitude displayed a strong positive correlation with the conditioned change in the stance H-reflex ( $p=0.004$; $r=+0.74$; slope $=+0.40$; intercept $=1.05)$. The regression indicates that, on the average, a given percentage change in the stance H-reflex was accompanied by a percentage change 0.4 times as large in soleus burst amplitude.

Figure 6 shows stance H-reflexes and right soleus bursts before and after conditioning from an HRdown rat and an HRup rat. After conditioning, both the stance $\mathrm{H}$-reflex and the soleus burst are smaller in the HRdown rat and larger in the HRup rat.

In contrast, H-reflex conditioning did not significantly affect the durations of the right and left soleus bursts nor the amplitude of the left soleus burst. Furthermore, conditioning did not appear to affect step-cycle duration or right/left symmetry. Because treadmill speed for each rat was the same before and after conditioning, these results also indicate that conditioning did not affect step-cycle length.

In the two unsuccessful HRdown rats that showed large increases in their stance H-reflexes (Fig. 5), right soleus burst amplitudes did not change (i.e., 103 and 104\% of control after conditioning, respectively).

\section{Discussion}

This study assessed the interactions between a new motor skill (an operantly conditioned decrease or increase in the right soleus H-reflex) and an old motor skill (locomotion). Both of these skills depend on soleus motoneurons and their response to primary afferent input. The difference is that the H-reflex depends almost entirely on soleus motoneurons and their primary afferent response, whereas locomotion depends also on many other motoneuron populations and their responses to many different kinds of input.

\section{The effects of locomotion on H-reflex conditioning}

The data summarized in Figure 3 and illustrated in Figure 4 show that successful $\mathrm{H}$-reflex conditioning was still evident during locomotion. In both HRdown and HRup rats, the locomotor $\mathrm{H}$-reflexes during stance and swing exhibited changes comparable with those of the conditioning $\mathrm{H}$-reflexes. The presence of these changes did not depend on the level of background EMG or on M-response size. As Figure 4 illustrates, the H-reflex changes were evident with higher and lower levels of background EMG and with larger and smaller $M$ responses. This persistence contrasts with the H-reflex changes that occur with a switch from standing to walking or from walking to running (Capaday and Stein, 1987; Stein, 1995; Faist et al., 1996). Unlike these situations, in which the H-reflex changes markedly when the concurrent task changes, the effects of conditioning on the H-reflex did not disappear when the rat began to walk.

This persistence of the effects of conditioning on the H-reflex is consistent with the slow time course of H-reflex conditioning [both during its initial development and during its reversal by exposure to the opposite mode (Wolpaw et al., 1986; Chen and Wolpaw, 1996)]. The time course implies that the motoneuron plasticity and other spinal cord plasticity that underlies the $\mathrm{H}$-reflex change develops gradually over days and weeks and thus would not be expected to simply disappear when the rat begins to walk and reappear when it stops. As reviewed in detail previously (Wolpaw, 2001; Wolpaw and Tennissen, 2001), H-reflex change is associated with changes in motoneuron firing threshold and conduction velocity, in F- and C-terminal populations on the motoneuron and probably in spinal interneurons and supraspinal regions as well. For example, the changes in motoneuron threshold and conduction velocity may be best explained by a positive shift in the activation voltage of sodium channels throughout the motoneuron, possibly mediated by protein kinase C (Carp and Wolpaw, 1994; Halter et al., 1995). This change would be unlikely to appear or disappear as the rat shifted from one behavior to another. On the other hand, it was theoretically possible that the effect of this plasticity on soleus response to primary afferent input would be blocked during locomotion by a compensatory change in presynaptic inhibition (i.e., a decrease in presynaptic inhibition during locomotion in HRdown rats and an increase in HRup rats) and thus that the conditioned change in 
CONDITIONING H-REFLEX

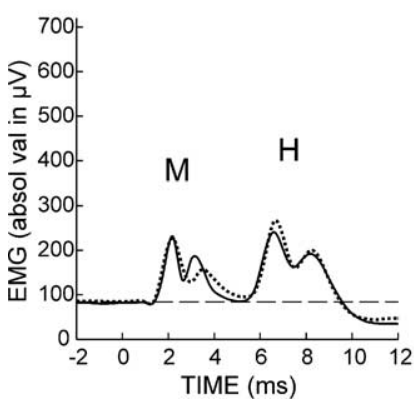

STANCE H-REFLEX

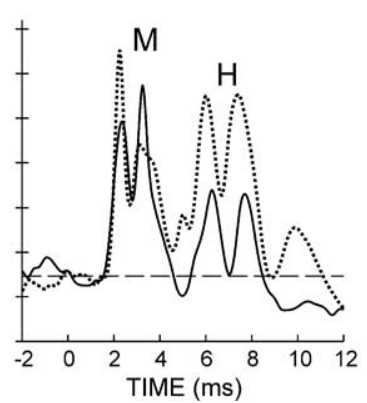

SWING H-REFLEX

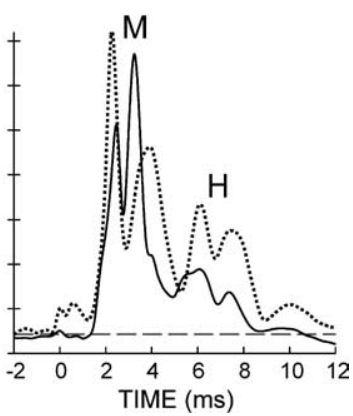

Figure 5. Conditioning and locomotor H-reflexes before (solid) and after (dotted) conditioning from an unsuccessful HRdown rat. The conditioning $\mathrm{H}$-reflexes are each the average of a single day (at least 4000 trials), and the locomotor $\mathrm{H}$-reflexes are each the average of 56-371 trials obtained during the treadmill session. Although the conditioning H-reflex is unchanged after conditioning, the stance and swing $\mathrm{H}$-reflexes are markedly increased. absol val, Absolute value.

the H-reflex would disappear. However, the data summarized in Figure 3 indicate that this did not happen. Rather, the H-reflex change remained evident during locomotion.

\section{The effects of H-reflex conditioning on locomotion}

Although the conditioned change in the H-reflex was not affected by locomotion, it was quite possible that locomotion would be affected by the conditioned change in the H-reflex. Primary afferent input contributes substantially to the locomotor burst that supports stance. It is estimated to be responsible for 35\% of the ankle-extensor force in decerebrate cats (Stein et al., 2000), 23\% of the triceps surae force in spinalized cats given clonidine (Bennett et al., 1996), and 30-60\% of the soleus burst in normal humans (Yang et al., 1991). Thus, the change in the response to this input caused by H-reflex conditioning might be expected to change, or even impair, locomotion. Right-leg stance might collapse in HRdown rats, whereas in HRup rats, it might be characterized by increased plantarflexion of the ankle. Right-leg stance might become briefer in HRdown rats and longer in HRup rats, leading to asymmetries in the step cycle. (In addition, locomotor adjustments to perturbations such as an unexpected obstacle or a sudden change in the slope of the walking surface might be compromised.) However, the present data provide no evidence for gross disturbances in normal locomotion caused by H-reflex conditioning. As Table 1 summarizes, neither HRdown nor HRup conditioning appeared to affect step-cycle or soleus burst durations or right/left symmetry.

Nevertheless, H-reflex conditioning did have the expected effects on the strength of the right soleus burst. As shown in Table 1 and illustrated in Figure 6, conditioned change in H-reflex size was strongly correlated with change in burst amplitude. Furthermore, the magnitude of the burst change (i.e., $40 \%$ of the magnitude of the H-reflex change) is consistent with data on the contribution of primary afferent input to the burst (Yang et al., 1991; Bennett et al., 1996; Stein et al., 2000). This finding indicates that the impact of H-reflex conditioning on locomotion was not prevented by compensatory change in muscle spindle sensitivity (i.e., increased in HRdown rats and decreased in HRup rats) or compensatory changes in other synaptic inputs to the motoneuron (i.e., increased excitation in HRdown rats and decreased excitation in HRup rats).

H-reflex conditioning had no significant effect on the left soleus burst, which was unchanged or somewhat smaller in both HRdown and HRup rats. This is consistent with primate data indicating that unilateral $\mathrm{H}$-reflex conditioning has little effect on the contralateral H-reflex of the awake animal (Wolpaw et al., 1993) [although it does change the contralateral spinal cord (Wolpaw and Lee, 1989)].

In sum, the plasticity produced by H-reflex conditioning did affect CNS function during locomotion, and this effect was not counteracted by changes in muscle spindle sensitivity, presynaptic inhibition, or other synaptic inputs. Conditioning of the right soleus H-reflex changed the response of the muscle to primary afferent input during locomotion and also changed the amplitude of soleus activation during stance. Nevertheless, these unilateral changes did not appear to affect step-cycle duration or symmetry. The absence of such effects has several possible explanations. First, because the soleus is only one, and not the largest, of the muscles providing plantarflexion, the change in the soleus burst caused by H-reflex conditioning might not have been sufficient to produce a detectable effect on the step cycle. Although this is certainly possible, the data on the effects of soleus $\mathrm{H}$-reflex conditioning on the gastrocnemius $\mathrm{H}$-reflex suggest that the gastrocnemius locomotor burst was similarly affected to some extent. This makes it less likely that the effects of conditioning on the locomotor burst were simply too small to affect the step cycle. (Furthermore, as noted below, preliminary data suggest that soleus H-reflex conditioning affects locomotion in spinal cord-injured rats and thus imply that it has significant kinematic impact.)

Second, the change in the soleus (and probably gastrocnemius) locomotor bursts might have induced automatic changes in the activity of other muscles. For example, in HRdown rats, the increase in ankle dorsiflexion caused by the reduced soleus burst might have produced proprioceptive input that increased activity in right quadriceps muscles and thereby increased knee extension during stance so as to balance out the increased ankle dorsiflexion and maintain body height. Such automatic compensatory changes would be constantly present as the rat moved about the cage and would therefore be expected to be accompanied by additional activity-dependent plasticity. This possibility is consistent with evidence that changes in muscle activation produced by nerve transections induce compensatory changes in other muscles (Whelan and Pearson, 1997; Pearson et al., 1999; Bouyer et al., 2001). In the present case, the additional compensatory plasticity would ensure preservation of normal locomotion, despite the fact that the pattern of muscle activations underlying locomotion was different from the pattern before H-reflex conditioning.

Such compensatory plasticity may help account for the multisite spinal and supraspinal plasticity associated with H-reflex conditioning, particularly those aspects of it that do not seem to underlie the operantly conditioned change in H-reflex size. For example, $\mathrm{H}$-reflex conditioning in monkeys is accompanied by a large decrease in the amplitude of heteronymous (but not homonymous) primary afferent EPSPs that has no obvious relationship to H-reflex change (Carp and Wolpaw, 1994, 1995). Furthermore, although down-conditioning in monkeys does not change the contralateral H-reflex of the awake animal, it greatly increases the contralateral motoneuron response to primary afferent input when the spinal cord is isolated from supraspinal influence (Wolpaw and Lee, 1989). In a perhaps related manner, 
Table 1. Effects of conditioning on locomotion

\begin{tabular}{|c|c|c|c|c|c|c|}
\hline & \multicolumn{2}{|c|}{ Right soleus burst } & \multicolumn{2}{|c|}{ Left soleus burst } & \multicolumn{2}{|l|}{ Step cycle } \\
\hline & Amplitude & Duration & Amplitude & Duration & Duration & Symmetry \\
\hline HRdown rats & $0.88(0.06)$ & $1.08(0.04)$ & $0.83(0.14)$ & $1.10(0.04)$ & $1.05(0.02)$ & $1.05(0.03)$ \\
\hline HRup rats & $1.29(0.18)$ & $1.09(0.04)$ & $0.93(0.21)$ & $1.03(0.01)$ & $0.99(0.03)$ & $1.03(0.01)$ \\
\hline Correlation & $p=0.004$ & NS & NS & NS & NS & NS \\
\hline
\end{tabular}

Average ( \pm SE) right and left soleus burst amplitudes and durations and step-cycle duration and right/left symmetry after conditioning for successful HRdown and HRup rats (expressed in terms of their control values) and the correlation between changes in them and the change in stance H-reflex (with the H-reflex change expressed in terms of the control value of soleus burst amplitude). NS, No significant correlation ( $p>0.05$ ).

HRdown RAT
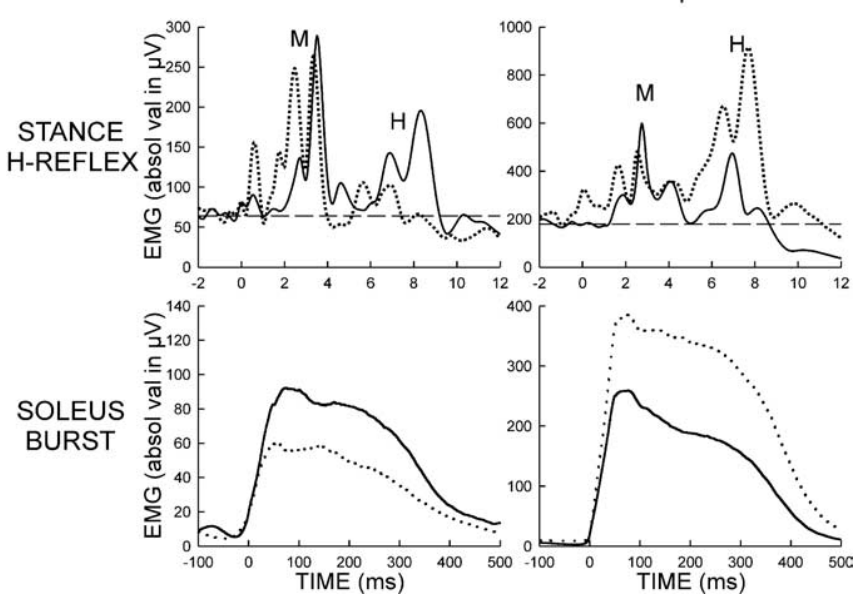

Figure 6. Stance H-reflexes and right soleus locomotor bursts before (solid) and after (dotted) conditioning from an HRdown and an $\mathrm{HRup}$ rat. The stance $\mathrm{H}$-reflexes are each the average of 109-166 trials, and the stance bursts are each the average of 131-462 bursts obtained during the treadmill session. After conditioning, both the stance $\mathrm{H}$-reflex and the soleus burst are smaller in the HRdown rat and larger in the HRup rat. absol val, Absolute value.

transection of the corticospinal tract in successful HRdown rats leads over $10 \mathrm{~d}$ to an H-reflex that is significantly larger than the control H-reflex before down-conditioning (Chen and Wolpaw, 2002). Such plasticity, which is inexplicable when viewed simply in terms of H-reflex conditioning, may ensure that the change in muscle response to primary afferent input caused by H-reflex conditioning does not change step duration, length, or symmetry or impair responses to obstacles, grade changes, or other perturbations.

\section{The etiology of conditioning failure}

Four HRdown rats failed to change the conditioning H-reflex (i.e., it remained within $20 \%$ of its control value). In two of these four, the locomotor H-reflexes were also not changed by conditioning. However, in the other two, the locomotor H-reflexes were much larger after conditioning, despite the fact that neither the conditioning H-reflexes nor the soleus locomotor bursts were changed. As a wholly unexpected finding, this remarkable increase is comparable with the increased contralateral response seen in the isolated spinal cord of HRdown monkeys (Wolpaw and Lee, 1989) and to the larger-than-control H-reflexes that corticospinal tract transection produces in successful HRdown rats (Chen and Wolpaw, 2002) or that the down-conditioning protocol produces in rats with sensorimotor cortex ablations (Chen et al., 2004).

Together, these unexpected and apparently inexplicable results imply that the final effect of $\mathrm{H}$-reflex conditioning reflects the combination of several activity-dependent processes. In successful rats, the combined effect is an appropriate H-reflex change, although in unsuccessful rats, the combined effect may be no change, or even an inappropriate change. Furthermore, the combined effect may differ depending on the situation in which it is assessed. For example, in the unsuccessful HRdown rat in Figure 5, the H-reflex elicited in the context of the steady-state muscle activity of the conditioning protocol is little changed, whereas the H-reflex elicited during or between the soleus locomotor bursts is greatly increased.

\section{Analysis of skill interactions}

The results suggest that it is not sufficient to address the interactions of new and old motor skills in terms of overarching concepts such as memory consolidation, which are not readily interfaced with the complexity of the neuronal and synaptic mechanisms that contribute to each skill. Locomotion is a skill that comprises activity-dependent plasticity at multiple sites. Any new skill that affects one or more of these sites is likely to affect locomotion in some way. The nature of this effect and its ultimate impact on both skills are issues that will yield only to detailed mechanistic investigations, and these are facilitated by simple models such as $\mathrm{H}$-reflex conditioning. It is from such studies that more adequate general concepts are likely to emerge. For example, by indicating that soleus $\mathrm{H}$-reflex conditioning affects how locomotion is produced, the present results suggest that locomotion never undergoes consolidation but rather is continually maintained by activity-dependent adaptive processes comparable with those responsible for its original acquisition.

\section{Possible applications of $\mathrm{H}$-reflex conditioning}

As noted above, the preservation of locomotion, despite the change in soleus activation and responsiveness produced by $\mathrm{H}$-reflex conditioning, is likely to reflect automatic adjustments by other muscles and possibly additional adaptive activitydependent plasticity, that is, plasticity that is triggered by disturbances that result from the soleus changes and serves to ensure continued normal locomotion.

However, when locomotion is already abnormal, as a result of spinal cord injury or another chronic disorder of supraspinal control, the same automatic adjustments and the same impetus for adaptation to eliminate the impact of $\mathrm{H}$-reflex conditioning may be impaired or absent. In such pathological situations, H-reflex conditioning might be used to substantially modify locomotion or even to restore more effective locomotion. For example, when stance during locomotion is inadequate or inconsistent, the increase in motoneuron response to primary afferent input caused by H-reflex up-conditioning might help to restore more effective or consistent stance. Spinal reflex conditioning is possible in people with partial spinal cord injuries (Segal and Wolf, 1994), and preliminary studies in spinal cord-injured rats suggest that such conditioning can improve locomotion (Chen et al., 2005a).

Activity-dependent plasticity in the spinal cord can be induced by sensory input from the periphery or by descending input from the brain (for review, see Wolpaw and Tennissen, 
2001). The sensory input created by assisted treadmill locomotion can induce spinal cord plasticity and thereby improve locomotion (Shurrager and Dykman, 1951; Lovely et al., 1986; Barbeau and Rossignol, 1987; de Leon et al., 2002; Dietz and Harkema, 2004). The descending input created by reflex conditioning might also be used to change the spinal cord so as to improve function. Furthermore, as methods for inducing spinal cord regeneration after injury are developed (Schwab and Bartholdi, 1996; McTigue et al., 2000; Selzer, 2003), H-reflex conditioning and conditioning of other simple reflexes (Chen et al., 2005b) might provide flexible and precise methods for reeducating the regenerated spinal cord so as to maximize recovery of function.

\section{Conclusions}

Although the new skill of H-reflex conditioning and the old skill of locomotion both depend on motoneuron response to primary afferent input, they do not appear to interfere with each other: conditioned H-reflex change is still evident during locomotion, and step-cycle duration, length, and symmetry are unchanged. However, H-reflex conditioning does affect how locomotion is produced: it changes soleus burst amplitude and probably induces compensatory changes in the activity of other muscles. Locomotion after H-reflex conditioning is indistinguishable in step-cycle duration, length, and symmetry from locomotion before, but the CNS activity underlying it is different. This altered activity may involve additional activity-dependent plasticity that contributes to the complex plasticity associated with H-reflex conditioning. Operant conditioning of $\mathrm{H}$-reflexes or other spinal reflexes may be able to reduce the functional deficits associated with spinal cord injury or other disorders of motor control.

\section{References}

Baldissera F, Hultborn H, Illert M (1981) Integration in spinal neuronal systems. In: Handbook of physiology, Sec I, The nervous system, Vol II, Motor control, Pt I (Brooks VB, ed), pp 509-595. Baltimore: Williams and Wilkins.

Barbeau H, Rossignol S (1987) Recovery of locomotion after chronic spinalization in the adult cat. Brain Res 412:84-95.

Bennett DJ, De Serres SJ, Stein RB (1996) Gain of the triceps surae stretch reflex in decerebrate and spinal cats during postural and locomotor activities. J Physiol (Lond) 496:837-850.

Blazquez PM, Hirata Y, Heiney SA, Green AM, Highstein SM (2003) Cerebellar signatures of vestibulo-ocular reflex motor learning. J Neurosci 23:9742-9751.

Bouyer LJ, Whelan PJ, Pearson KG, Rossignol S (2001) Adaptive locomotor plasticity in chronic spinal cats after ankle extensors neurectomy. J Neurosci 21:3531-3541.

Brown WF (1984) The physiological and technical basis of electromyography. Boston MA: Butterworths.

Burghardt PR, Fulk LJ, Hand GA, Wilson MA (2004) The effects of chronic treadmill and wheel running on behavior in rats. Brain Res 1019:84-96.

Caithness G, Osu R, Bays P, Chase H, Klassen J, Kawato M, Wolpert DM, Flanagan JR (2004) Failure to consolidate the consolidation theory of learning for sensorimotor adaptation tasks. J Neurosci 24:8662-8671.

Capaday C, Stein RB (1987) Difference in the amplitude of the human soleus $\mathrm{H}$ reflex during walking and running. J Physiol (Lond) 392:513-522.

Carey MR, Lisberger SG (2002) Embarrassed, but not depressed: eye opening lessons for cerebellar learning. Neuron 35:223-226.

Carp JS, Wolpaw JR (1994) Motoneuron plasticity underlying operantly conditioned decrease in primate H-reflex. J Neurophysiol 72:431-442.

Carp JS, Wolpaw JR (1995) Motoneuron properties after operantly conditioned increase in primate H-reflex. J Neurophysiol 73:1365-1373.

Carp JS, Chen XY, Sheikh H, Wolpaw JR (2001) Operant conditioning of rat H-reflex affects motoneuron axonal conduction velocity. Exp Brain Res 136:269-273

Carrier L, Brustein S, Rossignol S (1997) Locomotion of the hindlimbs after neurectomy of ankle flexors in intact and spinal cats. J Neurophysiol 77:1979-1993.

Chen XY, Wolpaw JR (1995) Operant conditioning of H-reflex in freely moving rats. J Neurophysiol 73:411-415.

Chen XY, Wolpaw JR (1996) Reversal of H-reflex operant conditioning in the rat. Exp Brain Res 112:58-62.

Chen XY, Wolpaw JR (1997) Dorsal column but not lateral column transection prevents down-conditioning of $\mathrm{H}$ reflex in rats. J Neurophysiol 78:1730-1734.

Chen XY, Wolpaw JR (2002) Probable corticospinal tract control of spinal cord plasticity in the rat. J Neurophysiol 87:645-652.

Chen XY, Wolpaw JR, Jakeman LB, Stokes BT (1996) Operant conditioning of H-reflex in spinal cord-injured rats. J Neurotrauma 13:755-766.

Chen XY, Wolpaw JR, Jakeman LB, Stokes BT (1999) Operant conditioning of H-reflex increase in spinal cord-injured rats. J Neurotrauma 16:175-186.

Chen XY, Feng-chen KC, Chen L, Stark DM, Wolpaw JR (2001a) Shortterm and medium-term effects of spinal cord tract transections on soleus H-reflex in freely moving rats. J Neurotrauma 18:313-327.

Chen XY, Chen L, Wolpaw JR (2001b) Time course of H-reflex conditioning in the rat. Neurosci Lett 302:85-88.

Chen XY, Carp JS, Chen L, Wolpaw JR (2002a) Corticospinal tract transection prevents operantly conditioned H-reflex increase in rats. Exp Brain Res 144:88-94.

Chen XY, Chen L, Wolpaw JR, Jakeman LB (2002b) Corticospinal tract transection reduces H-reflex circadian rhythm in rats. Brain Res 942:101-108.

Chen XY, Chen L, Wolpaw JR (2003) Conditioned H-reflex increase persists after transection of the main corticospinal tract in rats. J Neurophysiol 90:3572-3578.

Chen XY, Chen L, Wolpaw JR (2004) Contralateral sensorimotor cortex ablation in rats impairs $\mathrm{H}$-reflex up-conditioning and reverses the effect of down-conditioning. Soc Neurosci Abstr 30:417.4.

Chen Y, Chen XY, Jakeman LB, Chen L, Stokes BT, Wolpaw JR (2005a) Reeducating the injured spinal cord by operant conditioning of a reflex pathway. Soc Neurosci Abstr, in press.

Chen L, Chen XY, Liu RL, Wolpaw JR (2005b) Operant conditioning of reciprocal inhibition in freely moving rats. Soc Neurosci Abstr, in press.

Cohen TE, Kaplan SW, Kandel ER, Hawkins RD (1997) A simplified preparation for relating cellular events to behavior: mechanisms contributing to habituation, dishabituation, and sensitization of the Aplysia gillwithdrawal reflex. J Neurosci 17:2886-2899.

Compact Oxford English Dictionary (1993) Ed 2 (Weiner ESC, Simpson JA, eds), p 1782. Oxford: Oxford UP.

de Leon RD, Kubasak MD, Phelps PE, Timoszyk WK, Reinkensmeyer DJ, Roy RR, Edgerton VR (2002) Using robotics to teach the spinal cord to walk. Brain Res Brain Res Rev 40:267-273.

Dietz V, Harkema SJ (2004) Locomotor activity in spinal cord-injured persons. J Appl Physiol 96:1954-1960.

Evatt ML, Wolf SL, Segal RL (1989) Modification of human spinal stretch reflexes: preliminary studies. Neurosci Lett 105:350-355.

Faist M, Dietz V, Pierrot-Deseilligny E (1996) Modulation, probably presynaptic in origin, of monosynaptic Ia excitation during human gait. Exp Brain Res 109:441-449.

Garcia KS, Steele PM, Mauk MD (1999) Cerebellar cortex lesions prevent acquisition of conditioned eyelid responses. J Neurosci 19:10940-10947.

Goedert KM, Willingham DB (2002) Patterns of interference in sequence learning and prism adaptation inconsistent with the consolidation hypothesis. Learn Mem 9:279-292.

Grillner S (1981) Control of locomotion in bipeds, tetrapods and fish. In: Handbook of physiology, Sec 1, The nervous system, Vol 2, Motor control (Brooks VB, ed), pp 1179-1236. Bethesda, MD: American Physiological Society.

Halter JA, Carp JS, Wolpaw JR (1995) Operantly conditioned motoneuron plasticity: possible role of sodium channels. J Neurophysiol 73:867-871.

Hansel C, Linden DJ, DíAngelo E (2001) Beyond parallel fiber LTD: the diversity of synaptic and non-synaptic plasticity in the cerebellum. Nat Neurosci 4:467-475.

Henneman E, Mendell LM (1981) Functional organization of motoneuron pool and inputs. In: Handbook of physiology, Sec 1, The nervous system, Vol 2, Motor control (Brooks VB), pp 423-507. Bethesda, MD: American Physiological Society. 
King DAT, Krupa DJ, Foy MR, Thompson RF (2001) Mechanisms of neuronal conditioning. Int Rev Neurobiol 45:313-337.

Krakauer JW, Ghilardi MF, Ghez C (1999) Independent learning of internal models for kinematic and dynamic control of reaching. Nat Neurosci 2:1026-1031.

Lieb JR, Frost WN (1997) Realistic simulation of the Aplysia siphonwithdrawal reflex circuit: roles of circuit elements in producing motor output. J Neurophysiol 77:1249-1268.

Lisberger SG (1998) Physiologic basis for motor learning in the vestibuloocular reflex. Arch Otolaryngol Head Neck Surg 119:43-48.

Lovely RG, Gregor RJ, Roy RR, Edgerton VR (1986) Effects of training on the recovery of full-weight-bearing stepping in the adult spinal cat. Exp Neurol 92:421-435.

Magladery JW, Porter WE, Park AM, Teasdall RD (1951) Electrophysiological studies of nerve and reflex activity in normal man. IV. The twoneuron reflex and identification of certain action potentials from spinal roots and cord. Bull Johns Hopkins Hosp 88:499-519.

Matthews PBC (1972) The reflex actions of the muscle receptors. In: Mammalian muscle receptors and their central actions (Davson H, Greenfield ADM, Whittam R, Brindley GS, eds), pp 319-409. Baltimore: Williams and Wilkins.

McTigue DM, Popovich PG, Jakeman LB, Stokes BT (2000) Strategies for spinal cord injury repair. Prog Brain Res 128:3-8.

Medina JF, Nores WL, Ohyama T, Mauk MD (2000) Mechanisms of cerebellar learning suggested by eyelid conditioning. Curr Opin Neurobiol 10:717-724.

Medina JF, Repa JC, Mauk MD, LeDoux JE (2002) Parallels between cerebellum- and amygdala-dependent conditioning. Nat Rev Neurosci 3:122-131.

Pearson KG (1993) Common principles of motor control in vertebrates and invertebrates. Annu Rev Neurosci 16:265-297.

Pearson KG, Fouad K, Misiaszek JE (1999) Adaptive changes in motor activity associated with functional recovery following muscle denervation in walking cats. J Neurophysiol 82:370-381.

Schwab ME, Bartholdi D (1996) Degeneration and regeneration of axons in the lesioned spinal cord. Physiol Rev 76:319-370.

Segal RL, Wolf SL (1994) Operant conditioning of spinal stretch reflex in patients with spinal cord injuries. Exp Neurol 130:202-213.

Selzer ME (2003) Promotion of axonal regeneration in the injured CNS. Lancet Neurol 2:157-166.

Shadmehr R, Holcomb HH (1997) Neural correlates of motor memory consolidation. Science 277:821-825.

Shurrager PS, Dykman RA (1951) Walking spinal carnivores. J Comp Physiol Psychol 44:252-262.
Stein RB (1995) Presynaptic inhibition in humans. Prog Neurobiol 47:533-544.

Stein RB, Misiaszek JE, Pearson KG (2000) Functional role of muscle reflexes for force generation in the decerebrate walking cat. J Physiol (Lond) 525:781-791.

Thompson RF, Bao S, Chen L, Cipriano BD, Grethe JS, Kim JJ, Thompson JK, Tracy JA, Weninger MS, Krupa DJ (1997) Associative learning. Int Rev Neurobiol 41:151-189.

van Alphen AM, De Zeeuw CI (2002) Cerebellar LTD facilitates but is not essential for long-term adaptation of the vestibulo-ocular reflex. Eur J Neurosci 16:486-490.

Walker MP, Brakefield T, Hobson JA, Stickgold R (2003) Dissociable stages of human memory consolidation and reconsolidation. Nature 425:616-620.

Whelan P, Pearson KG (1997) Plasticity in reflex pathways controlling stepping in the cat. J Neurophysiol 78:1643-1650.

Wigmore V, Tong C, Flanagan JR (2002) Visuomotor rotations of varying size and direction compete for a single internal model in motor working memory. J Exp Psychol Hum Percept Perform 28:447-457.

Wolpaw JR (1987) Operant conditioning of primate spinal reflexes: the H-reflex. J Neurophysiol 57:443-459.

Wolpaw JR (2001) Spinal cord plasticity in the acquisition of a simple motor skill. In: Spinal cord plasticity alterations in reflex function (Patterson MM, Grau JW, eds), pp 101-125. Boston: Kluwer Academic.

Wolpaw JR (2002) Memory in neuroscience: rhetoric versus reality. Behav Cogn Neurosci Rev 1:130-163.

Wolpaw JR, Lee CL (1989) Memory traces in primate spinal cord produced by operant conditioning of H-reflex. J Neurophysiol 61:563-572.

Wolpaw JR, Tennissen AM (2001) Activity-dependent spinal cord plasticity in health and disease. Annu Rev Neurosci 24:807-843.

Wolpaw JR, Braitman DJ, Seegal RF (1983a) Adaptive plasticity in primate spinal stretch reflex: initial development. J Neurophysiol 50:1296-1311.

Wolpaw JR. Seegal RF. O'Keefe JA (1983b) Adaptive plasticity in primate spinal stretch reflex: behavior of synergist and antagonist muscles. J Neurophysiol 50:1312-1319.

Wolpaw JR, O’Keefe JA, Noonan PA, Sanders MG (1986) Adaptive plasticity in primate spinal stretch reflex: persistence. J Neurophysiol 55:272-279.

Wolpaw JR, Herchenroder PA, Carp JS (1993) Operant conditioning of the primate H-reflex: factors affecting the magnitude of change. Exp Brain Res 97:31-39.

Yang JF, Stein RB, James KB (1991) Contribution of peripheral afferents to the activation of the soleus H-reflex in walking in humans. Exp Brain Res 87:679-687. 